\section{Chinolon-Hypersensitivität identifizieren}

\author{
Chinolone können IgE-vermittelte Hypersensitivitätsreaktionen \\ auslösen. Hauttests sind nicht immer zuverlässig. In einer Studie \\ wurde deshalb die Brauchbarkeit von In-vitro-Tests als Diagnose- \\ methode für diese Arzneimittelallergien untersucht.
}

$\mathrm{D}$ ie Antibiotika Ciprofloxacin, Levofloxacin und Moxifloxacin sind Chinolone, die zu den Gyrasehemmer zählen. Bei der Einnahme kann es zu Hypersensitivitätsreaktionen wie Urtikaria und Anaphylaxie kommen, vor allem bei der Gabe von Moxifloxacin. Da Hauttests durch häufig vorkommende falsch-positive Ergebnisse nicht sehr zuverlässig sind, wurde die Eignung des Basophilenaktivierungstests (BAT) und des Sepharose-Radioimmunassays (RIA) als In-vitro-Testmethoden für den Nachweis einer Hypersensitivitätsreaktion untersucht.

38 Probanden mit bekannter allergischer Chinolon-Sofortreaktion wurden in die Studie aufgenommen. Die Reaktionen waren bei 24 Patienten unter Moxifloxacin, bei elf unter Ciprofloxacin und bei drei unter Levofloxacin aufgetreten. Die Sepharose-RIA war bei zwölf Patienten (31,6\%) und der BAT bei 27 $(71,1 \%)$ positiv. Im Sepharose-RIA reagierten die Seren von acht Patienten $(21,0 \%)$ positiv auf Ciprofloxacin, von sieben Patienten (18,4\%) auf Moxifloxacin und von sieben $(18,4 \%)$ auf Levofloxacin. Für den BAT traten entsprechende Positivresultate bei 23 (60,5\%), zwölf $(31,6 \%)$ und acht Patienten $(21 \%)$ auf.

Die Spezifität des Sepharose-RIA wurde per Inhibitionstest nachgewiesen. Für den Nachweis, dass die Basophilen- aktivierung tatsächlich durch IgE-Antikörper vermitteltet war, wurde der PI3K(Phosphatidylinositol-3-Kinase)Inhibitor Wortmannin verwendet. Dieser Kinaseinhibitor hemmt über eine Blockade der Histaminfreisetzung die Basophilenaktivierung bei Stimulation mit Anti-IgE und den verschiedenen Chinolonen.

Fazit: IgE-vermittelte Chinolon-Hypersensitivitätsreaktionen können vor allem bei Anaphylaxiepatienten, bei denen keine Provokationstestung möglich ist, durch die Sepharose-RIA und vor allem den BAT diagnostiziert werden. Die Sensitivität der beiden Verfahren hängt vom jeweiligen Chinolon ab, ein Phänomen, das auch von den Benzylpenicillinen bekannt ist.

Dr. Barbara Kreutzkamp

Aranda A et al. In vitro evaluation of IgEmediated hypersensitivity reaction to quinolones. Allergy 2011; 66: 247-54

\section{Allergische Reaktionen: ein unterschätztes Narkoserisiko}

\author{
Jede Narkose birgt Risiken. Doch einem Risiko wurde bislang zu \\ wenig Beachtung geschenkt: Allergien auf Narkosemittel. Mehr \\ Patienten als angenommen erleiden einen solchen Zwischenfall, \\ wie ein aktuelle Studie aus Frankreich zeigte.
}

E twa 100 allergisch bedingte Zwischenfälle kommen auf eine Million Narkosen. Das ist absolut gesehen nicht viel, aber deutlich mehr als die bislang angenommenen 77 auf eine Million. Diese Zahlen wurden kürzlich von französischen Wissenschaftlern publiziert. Sie griffen für ihre Untersuchung auf drei nationale Datenbanken (GERAP National Register, French Pharmacovigilance System, French National Survey of Anesthesia) zurück und werteten die Akten von 1.816 Patienten aus, die zwischen 1997 und 2004 eine IgE-vermittelte allergische Reaktion während der Narkose erlitten hatten.

Muskelrelaxanzien zeigten dabei das größte allergische Potenzial $(58,1 \%)$, gefolgt von Latex-haltigen Utensilien
(19,7\%) und Antibiotika (12,9\%). Was besonders erstaunt, ist der überproportional hohe Frauenanteil. Mit 155 pro eine Million Narkosen wartete das weibliche Geschlecht mit einer fast dreimal hö-

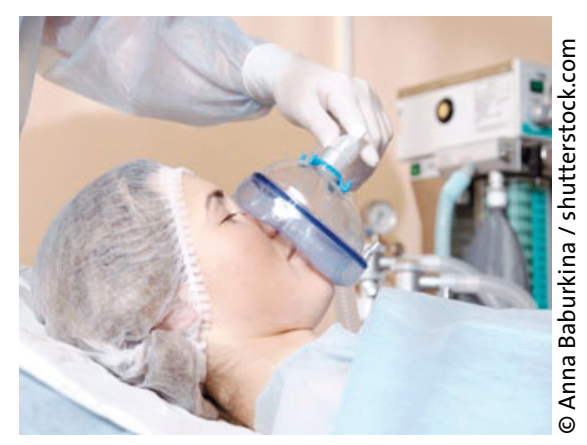

Frauen sind häufiger von allergisch bedingten $Z$ wischenfällen betroffen. heren Inzidenzrate auf als die Männer (55/1 Mio.). Betrachtet man ausschließlich die Zwischenfälle, die auf Muskelrelaxanzien zurückzuführen sind, spitzt sich die Situation nochmals zu. Bei Kindern hingegen lässt sich dieser geschlechtsspezifische Unterschied nicht feststellen. Daher vermuten die Autoren, dass weibliche Geschlechtshormone eine wichtige Rolle bei der Allergieentstehung spielen.

Fazit: Allergische Reaktionen sind ein ernst zu nehmendes Narkoserisiko und reichen von Hautausschlägen bis zum anaphylaktischen Schock. Frauen sind dabei stärker gefährdet als Männer. Vor allem bei Muskelrelaxanzien ist Vorsicht geboten. Die Autoren fordern deshalb, dass allergische Reaktionen künftig mit in die Beurteilung des NutzenRisiko-Verhältnisses verschiedener Narkoseregime einbezogen werden müssen.

Dr. Dagmar Kraus

Mertes PM et al. Anaphylaxis during anesthesia in France: An 8-year national survey. J Allergy Clin Immunol 2011; 128: 366-73 